\title{
Research on the application of virtual instrument technology in simulation training
}

\author{
Lei Ding ${ }^{1, *}$, Tao Pan $^{1}$, Liqing Qiao ${ }^{1}$, and Hongyu $\mathrm{Li}^{1}$ \\ ${ }^{1}$ Jiuquan Satellite Launch Centre, Jiuquan, Buge Yin'a Rila, Ejin Banner, Alxa League, China
}

\begin{abstract}
In orde to improve the space launch capability, the virtual instrument of the ground test equipment of the measuring system is studied by using the LaBVIEW technology in view of the present situation that there is no actual training equipment for measurement system in the emission field. A suit of virtual instrument for ground test equipment is developed to meet the requirements of daily post training. By analyzing the composition, function and working principle of the simulation training platform, this paper designs the system structure, simulation members, communication protocol and simulation flow of the simulation training platform, which can be used to complete the unit test, subsystem test, integrated test and fault disposal of the aircraft.The test operator can use the virtual instrument to have an intimate knowledge of the test flow, the operation steps, the test status, the fault disposal measures and other work contents. It can effectively improve the training level and test ability of the test operator in the emission field measurement system.
\end{abstract}

\section{Introduction}

Aiming at the present situation that there is no actual training equipment in the field measurement system. We research a virtual instrument for the ground test equipment of the launch site measurement system, in order to meet the higher requirements of high frequency and high density test missions, the test cycle, the quality of the testers and the operation ability. It can be to complete the unit test, sub-system test, integrated test and fault handling of the aircraft. The test operator can be familiar with the test flow, clear operation steps, clear test status, and master the work content of the fault handling measures by operating the virtual instrument. It can effectively improve the training level and comprehensive test ability.

\section{Composition, Function and Principle of Simulation Training Platform}

\subsection{Composition}

The simulation training platform mainly includes distribution console, simple telemetry station, image decryption machine and cipher machine. The distribution console is mainly

\footnotetext{
* Corresponding author: 18189377049@189.cn
} 
used to supply power to the aircraft measurement system on the ground; the simple telemetry station is mainly used to receive, demodulate, process and store the telemetry data of the aircraft; the image decryption machine is mainly used to display the image of the internal equipment of the aircraft; the cipher machine is mainly used to decrypt the telemetry data transmitted by the aircraft.

\subsection{Function}

The control logic of the simulation training platform is consistent with the real state, and should have the characteristics of practical operation and visualization. The implementation functions are as follows:

- The simulation members are used to simulate the parameters, display the states and control the key-press. It mainly includes the operating voltage/current parameters, leakage monitoring parameters, voltage regulation control, local control/distant control state conversion control and other parameters of the distribution console. It inclundes the display of left/right lock state, feed state, encryption and decryption state, uplink state parameter, receiver parameter demodulation state display, start-up disk control and other paramenters of the simple telemetry station.It also includes status display, boot control of the cipher machine.

- The simulation training platform has single mode and online mode. The single mode is mainly used to test the operator's single operation training, according to the single simulation member's operation manual, to achieve the purpose of skilled operation. And the online mode is mainly used for the system comprehensive training, according to the measurement system simulation training operation rules, to achieve the purpose of familiar with the test process, clear test steps, clear test status.

- The simulation training platform has working mode and fault mode. The working mode is mainly used in the test project of the launch field. The process flow control and state display are consistent with the real state, in order to receive, demodulate and process the data. The fault mode is mainly used to simulate the failure of wireless signal lockout, PCM signal encryption/decryption anomaly. The system command and operation personnel carry out on-site troubleshooting according to the requirements of the six-step method of emission field in order to improve the fault handling ability of the participants.

\subsection{Principle of Operation}

The distribution console provides $28 \mathrm{~V}$ supply voltage to the measurement system unit. The measurement system unit transmits many kinds of telemetry signals to the ground receiving antenna through the transmitting antenna. The received telemetry signal is transmitted to the simple telemetry station by the ground receiving antenna. The ciphertext data which is received and demodulated by the simple telemetry station is decrypted by the cipher machine. The decrypted plaintext data is sent to the simple telemetry station for processing and storage, and the image signal is sent to the image decryption machine by the simple telemetry station. The working principle of the ground simulation training platform of the measurement system is shown in figure 1 . 


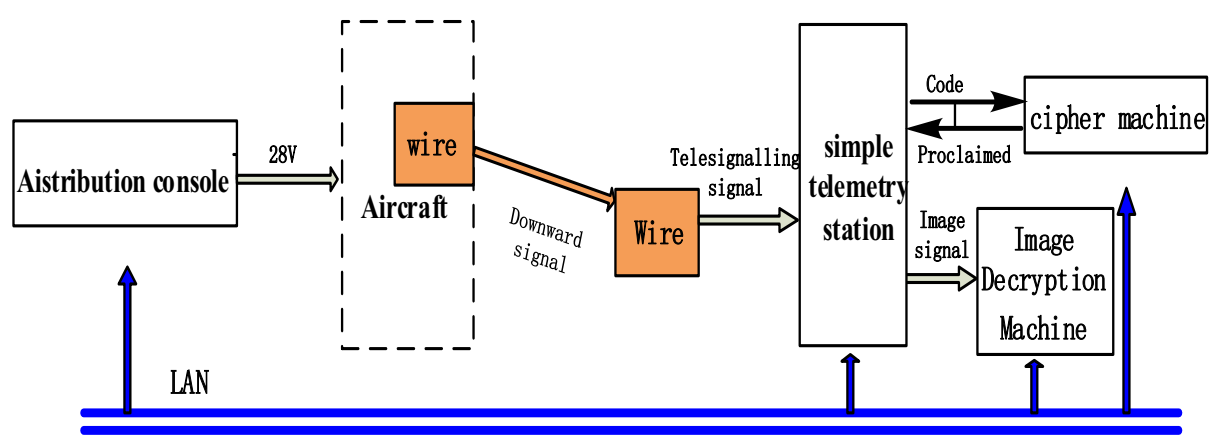

Fig. 1. The chematic diagram of the ground simulation taining platform.

\section{Design of Simulation Training Platform}

Based on the LabVIEW software system, the front panel and flow chart of the simulation members are constructed to realize the testing, processing and control functions of the simulation members.

\subsection{System Architecture}

The simulation training platform mainly simulates test project of the technical area in the launch field, and provides the virtual operation platform such as power supply, downlink signal locking / loss state display, cipher machine self-checking process state display and so on. The simulation training platform can adapt to the simulation of working mode and fault mode. It simulates the test flow, test state and operation steps of the ground equipment of the launch field measurement system, and provides a training platform for the test operators.

A LabVIEW simulation training platform is used in this design. The power-distribution console controls the simple telemetry station through the serial port to process and display the parameters of the power-off state of the measurement system unit.

\subsection{Simulation Component Design}

Simulation system composition includes power-distribution console, the simple telemetering station,image decryption machine and password machine.

- Design of the power-distribution console. It designs TCP communication function to realize signal communication with simple telemetry station. The console has two mode, the working mode and fault mode. The working mode is mainly uesed in the normal working state.It can display the parameter state, change the value of voltage and current string attribute by controlling the "power supply" control, and communicate with the simulation members of the simple telemetry station. The fault mode is mainly uesed in the fault state. In the fault mode,It does not design the TCP communication function between the simulation members of the simple telemetry station.

- Design of the simple telemetering station. It designes the TCP communication function to realize the send-receive function withduring the power-distribution console, the image decryption machine and the cipher machine .For example,it realizes the function of the signal lock, loses the lock, the secret, plaintext state conversion, the image display by changing the Boolean control; A simple telemetering station simulation member designs the working mode and the fault mode, controls the power-up and power-down with the Powersupply control, as well as the string attribute value and boolean attribute of various 
parameters in the state of signal lock, and receives or publishes the state and parameter information by the simulation thread of TCP communication response, and reacts various attributes and state changes in real time on the main interface.

- Design of Image Decryption Machine.It designs the Power-control to plus off, Boolean property changes.It achieves image display, state indication and other functions with the TCP communication .

- Design of Password Machine.It designs TCP communication function, uses the simple telemetry station to demodulate Boolean control property change, trigger cipher machine state 1 , state 2 , state 3 indicator Boolean control variable change. When the state $1,2,3$ boolean control are triggered, the variable value of the state 1,2,3 are true. When the state 1,2,3 boolean control are not triggered, the variable value of the state 1,2 are false, the variable value of state 3 is true.

\subsection{Simulation Member Communication Protocol Design}

This design uses LabVIEW communication software based on TCP protocol, the whole transmission process is as follows:

- The the power-distribution console sets up listening and waits for the simple telemetry station to connect through the machine number or IP address and port number; the simple telemetry station sets up listening through the machine number or IP address and port number, waits for the simple telemetry station to connect;

- The simple telemetry station sends out the connection request according to the address and port number IP the host computer of the simulation member of the distribution console; the cipher machine and the image decryption machine send out the connection request according to the address and port number IP the host computer of the simple telemetry station;

- After the power-distribution console and the simple telemetry station are connected, it communicates the TCP data through the read-write function; after the simple telemetry station,cipher machine and the image decryption machine are connected, the TCP data communication is carried out through the read-write function ;

- The power-distribution console is connected with the simple telemetry station to communicate the TCP data through the read-write function; after the simulation members of the simple telemetry station are connected with the simulation members of the cipher machine and the image decryption machine, the TCP data communication is carried out through the read-write function;

- Close communication.

\subsection{Simulation Process Design of Simulation Training Platform}

It realizes the simulation flow of simulation training platform in working mode and fault mode by LabVIEW design.

- Design of the working mode simulation flow. It simulates the work of power supply, wireless signal receive and demodulation,PCM signal encryption/decryption, image signal transmission and display, and realizes the test function of ground equipment of measurement system. the simulation workflow is shown in figure 2 . 


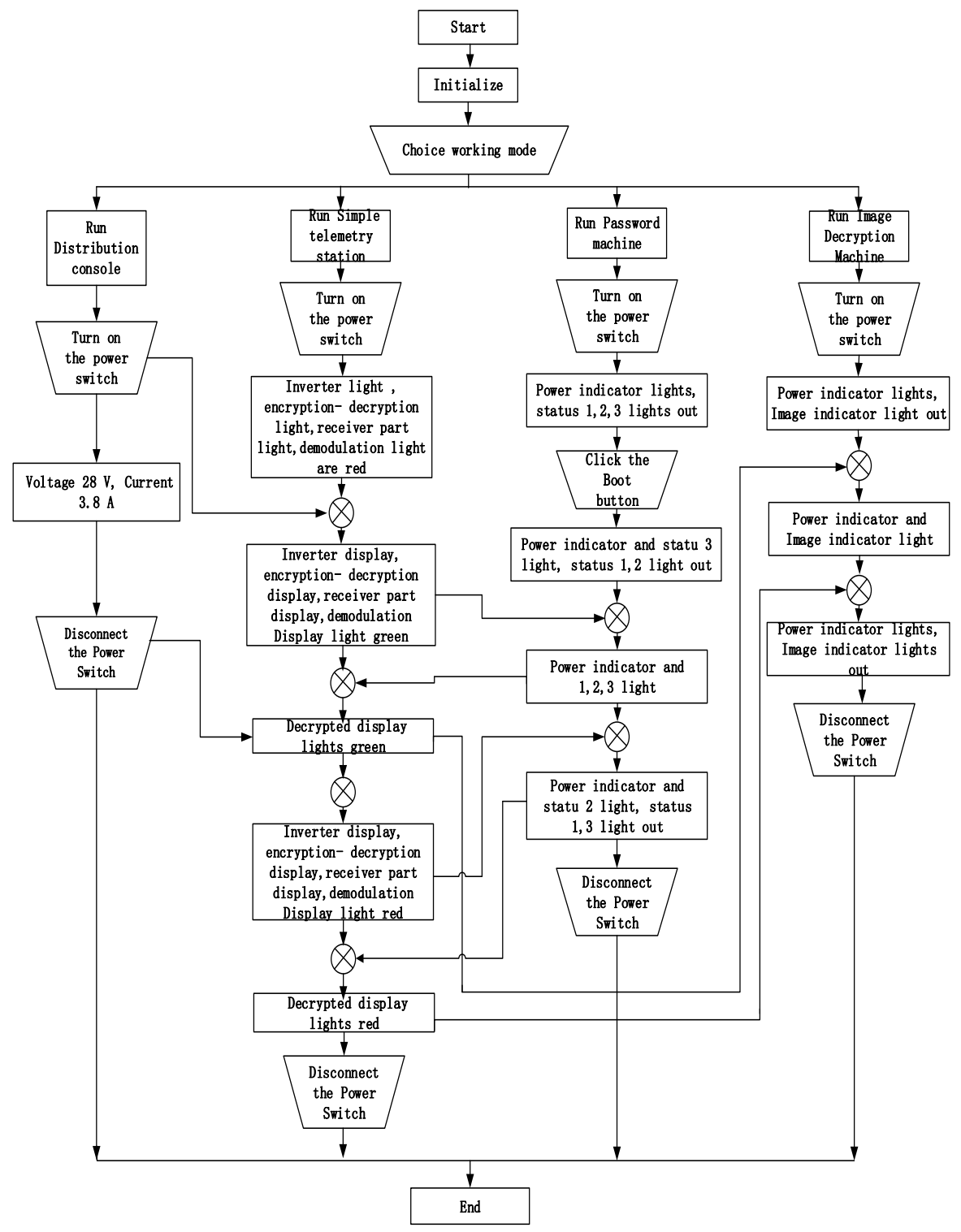

Fig. 2. Simulation Flowchart.

- Design of the fault mode simulation flow.It desines two fault modes, wireless signal lock failure mode and PCM signal encryption/decryption abnormal fault mode. The wireless signal lock failure mode simulates the working flow that the ground equipment of the measurement system can't receive the downlink signal of the aircraft normally, and the simple telemetry station can't demodulate the signal normally. The wireless signal loss fault mode is shown in figure 3. 


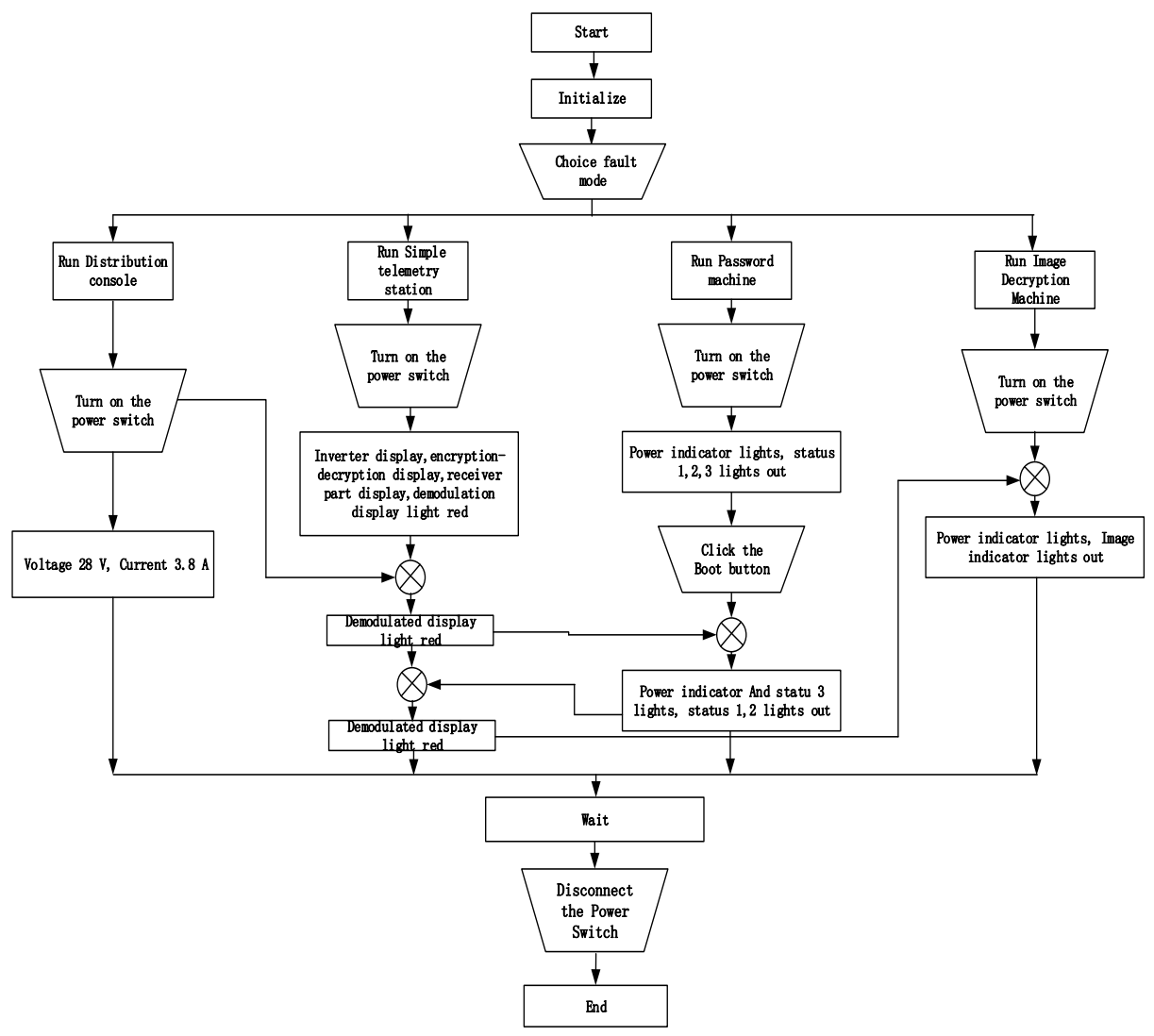

Fig. 3. Simulation Flow Diagram of Failure Mode of Wireless Signal Failure.

PCM signal encryption/decryption abnormal fault mode simulates the work flow that the cipher machine can't decrypt the data normally, and the ciphertext received and demodulated by the simple telemetry station is sent to the cipher machine for decryption, but the decryption indication signal can't be formed and not sent back to the processing terminal of the simple telemetry station. The PCM signal encryption / decryption abnormal fault mode is shown in figure 4. 


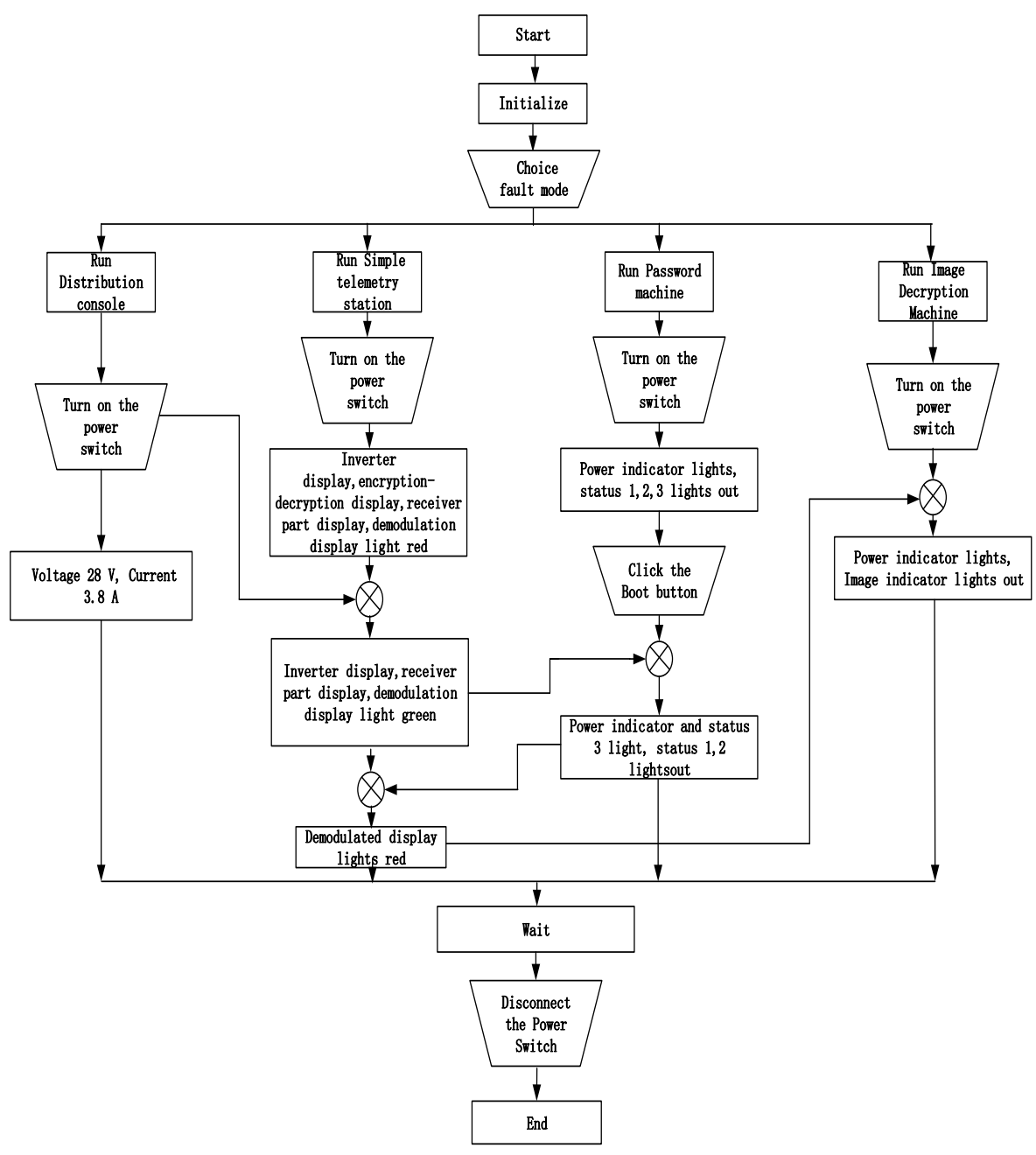

Fig. 4. PCM Simulation flow chart of abnormal fault mode of signal encryption/decryption.

\section{Conclusions}

This paper establishes a simulation training platform to integrate virtual technology with post training.It can not only make up for the problem that the measurement system in the launch field does not have the actual training equipment, but also improve the operator's operation skills, professional knowledge and the ability to deal with the failure. The continuous improvement of the comprehensive quality of the operators greatly shortens the test progress and development process, promotes the performance improvement, and provides a strong guarantee for improving the comprehensive test ability of the launch site.

\section{References}

1. R. Qizhen, Me and LabVIEW 2nd edition (Beijing University of Aeronautics and Astronautics Press Beijing, 2012)

2. X. Xiaodong, Common Functions and Programming Examples of the LabVIEW8.5 
(Electronic Industry Press, Beijing, China, 2009)

3. L. Jiangquan, 130 LabVIEW Virtual Instruments from Introduction to Measurement and Control (Electronic Industry Press, China, Beijing, 2013)

4. L. Xiangyang, Aerospace Testing Technology (National Defense Industry Press, China Beijing)

5. C. Shuxue, L. Xuan, LabVIEW 2nd Edition of the Treasure (Electronic Industry Press, Beijing, 2017). 